\title{
DSP-based digital FM demodulation for GMSK signals
}

\author{
B RAMAMURTHI, K GIRIDHAR and M A SRINIVAS \\ Department of Electrical Engineering, Indian Institute of Technology, Madras \\ 600 036, India
}

\begin{abstract}
Demodulation of Gaussian Minimum Shift Keying (GMSK) using a limiter-discriminator is a low complexity alternative to coherent demodulation. This so-called digital FM demodulation is followed by clock recovery, sampling, and thresholding. Conventionally, clock recovery is done in hardware, and matched filtering is usually not possible when the Gaussian pulse is wider than a bit duration. We propose a clock recovery technique based on discrete-time processing of the demodulated baseband signal. This technique couples very nicely with a new maximum likelihood sequence estimator for the data that uses a whitening filter followed by a Viterbi decoder. The entire detection algorithm can be implemented in an efficient manner on a Digital Signal Processor (DSP). Computer simulation results are presented to show that the new algorithm performs better than the conventional slicer by as much as $5.5 \mathrm{~dB}$.
\end{abstract}

Keywords. Viterbi decoding; digital FM receiver; GMSK demodulation.

\section{Introduction}

Gaussian Minimum Shift Keying (GMSK) is a popular bandwidth-efficient modulation scheme employed in many mobile and personal communication systems (GSM 1991; ETSI 1992). It permits the use of a variety of receivers, ranging from the coherent to the digital-FM type, each with a different complexity/performance trade-off. The transmitter can also be simplified depending on the type of receiver employed. A digital FM receiver, and a correspondingly simplified transmitter, is an extremely attractive low-complexity option. The digital FM modem has been known and used for many years now (Pawula 1981) but its performance has been quite poor when compared to the best possible receiver that one can implement, namely, a coherent receiver.

This paper describes an improved detection scheme for digital-FM reception of GMSK signals. The improvement is made feasible by the availability of Digital Signal Processors (DSPs). Since a DSP is almost always needed in modern communication systems for performing many tasks, it can be used for implementing the modem algorithm also. The improved algorithm is based entirely on digital signal processing techniques and requires only a small increase in complexity. Data clock synchronisation is achieved computationally using a correlator and interpolator. An improved bit error rate (BER) performance 
is obtained by replacing the slicer (based on simple thresholding) with a Viterbi detector which implements Maximum Likelihood Sequence Estimation (MLSE). This Viterbi Algorithm (VA) operates directly on Nyquist-rate samples of the demodulated baseband signal, and does not require a separate matched filter. Finally, it is shown that if an approximate noise-whitening filter is used prior to the VA, the BER performance can be further improved. $\S 2$ describes GMSK and its attributes, and in particular, the digital-FM demodulator for it. In $\S 3$, we discuss sampling of the received baseband signal, and a DSP-based clock recovery technique. The MLSE detector for the GMSK baseband signal is described in $\S 4$. An approximate whitening filter is also considered for the coloured noise at the demodulator output. The paper concludes with simulation results, some conclusions based on those results, and an outline of related future work.

\section{GMSK modulation and demodulation}

Wireless communication systems based on frequency division multiple access (FDMA) and/or time division multiple access (TDMA), require highly bandwidth-efficient modulation to minimise adjacent channel interference. Minimum Shift Keying (MSK) (Proakis 1989 , ch. 3) is a nonlinear modulation scheme which provides significantly superior outof-band power rejection when compared to linear modylation techniques with similar information (bit) rates. Further reduction of out-of-band power is possible by base-band pulse shaping. High bit-rate systems, such as those based on the GSM (GSM 1991) and DECT (ETSI 1992) standards, employ a Gaussian prefilter to meet the stringent specifications on adjacent channel interference. In this Gaussian MSK (Hirade \& Murota 1981) system, a trade-off between out-of-band power and intersymbol interference (ISI) is possible by varying the $B T$ product, where $B$ is the $3-\mathrm{dB}$ bandwidth of the Gaussian prefilter and $T$ is the symbol (bit) period.

The GMSK modulator is illustrated in figure 1, where the transmitted bandpass signal $s(t)$ is given by

$$
s(t)=A_{c} \cos \left(2 \pi f_{c} t+\phi(t)+\phi_{0}\right) .
$$

Here $A$ is the carrier amplitude, $f_{c}$ the carrier frequency, $\phi_{0}$ an arbitrary initial phase, and $\phi(t)$ a time-varying phase given by

$$
\phi(t)=\frac{\pi}{2} \int_{-\infty}^{t} m(\lambda) \mathrm{d} \lambda
$$

The signal $s(t)$ is thus an FM signal with message signal $m(t)$, where in turn $m(t)$ is given by

$$
m(t)=\sum_{k} I_{k} g(t-k T)
$$

The instantaneous frequency deviation of $s(t)$ is proportional to $m(t)$. $\operatorname{In}(3),\left\{I_{k}\right\}$ is a bipolar (random) information sequence and $g(t)$ represents the convolution of a rectangular pulse of duration $T$ seconds with the Gaussian prefilter whose impulse response is of the form $\beta \exp \left(-\alpha t^{2}\right)$. (Note that if the Gaussian filter is absent, we obtain MSK.) Although this implies that $g(t)$ is infinite in duration, $g(t)$ can be truncated to a finite duration in practice, and an appropriate delay introduced to make the filter causal.

For example, when $B T=0.5, g(t)$ can be taken to be zero for $t \notin\left[0, T_{g}\right]$ where $T_{g}=3 T$. Therefore, an ISI contribution from just one preceding and one succeeding 


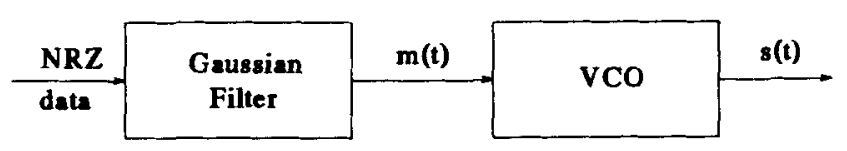

Figure 1. GMSK modulator

symbol distorts the transmitted pulse for the present symbol of $m(t)$. A sample waveform for this example is shown in figure 2, where $g(t)$ is for all practical purposes less than $3 T$ in duration. Moreover, even when $g(t)$ is truncated to a duration of $2 T$ (i.e., $T_{g}=2 T$ ), the maximum error by this approximation occurs at the peaks and valleys caused by the 010 and 101 bit patterns respectively, and is about $6 \%$.

Note also that it is not necessary to implement the GMSK modulator as shown in figure 1. It is generally not easy to implement a linear voltage controlled oscillator (VCO) with precise control of its sensitivity and centre-frequency. The modulated output $s(t)$ can be written in I-Q form using Rice's representation (Proakis 1993). The modulator can be thus implemented using a conventional balanced modulator with the in-phase and quadrature components as the baseband signals.

Both coherent and non-coherent demodulation of the GMSK signal are possible. A quadrature (I-Q) demodulation approach becomes obvious when we rewrite (1) as follows (assuming $\phi_{0}=0$ for convenience):

$$
s(t)=A \cos \phi(t) \cos 2 \pi f_{c} t-A \sin \phi(t) \sin 2 \pi f_{c} t
$$

where $A \cos \phi(t)$ and $A \sin \phi(t)$ are the in-phase and quadrature-phase signals, respectively. If the nominal variations in the carrier frequency (during modulation) are kept small, a coherent quadrature demodulator is possible as in conventional QPSK systems. However, when the instantaneous frequency is allowed to deviate significantly (for example, the DECT standard (ETSI 1992) allows as much as 30\% deviation from the ideal value), even differential detection of the I-Q data streams may not be possible. Such large errors in the instantaneous frequency are permitted in some standards in order to enable the use of a VCO-based GMSK modulator as shown in figure 1, which may not be able to maintain high accuracy. Such implementations can be very cost-effective, as for example a direct modulation transmitter, where the RF carrier is directly frequency-modulated. In fact, when the instantaneous phase is not maintained precisely according to (2), it is more appropriate to label the modulated signal as Gaussian Frequency-Shift Keying (GFSK) rather than GMSK.

A low-complexity alternative to the quadrature demodulator for GMSK is the simple FM limiter-discriminator followed by a sampler and detection device. Such a receiver is

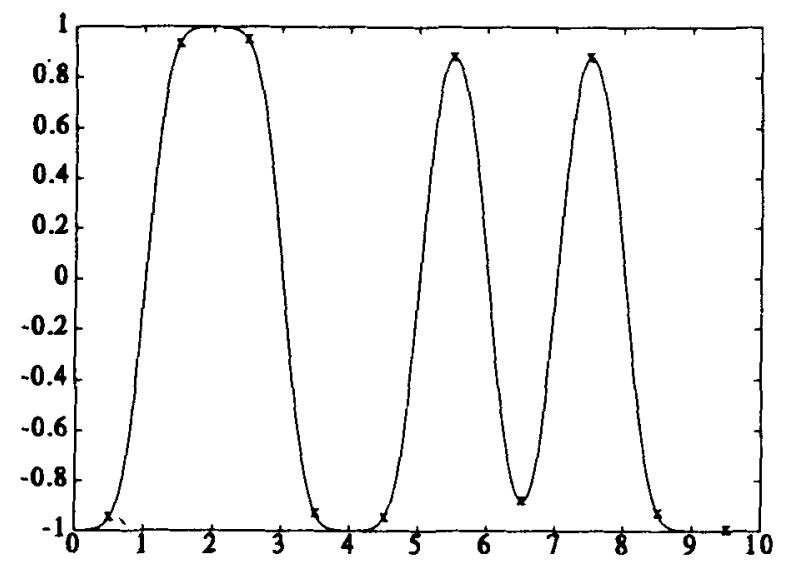

Figure 2. GMSK baseband signal for $B T=0.5$ 


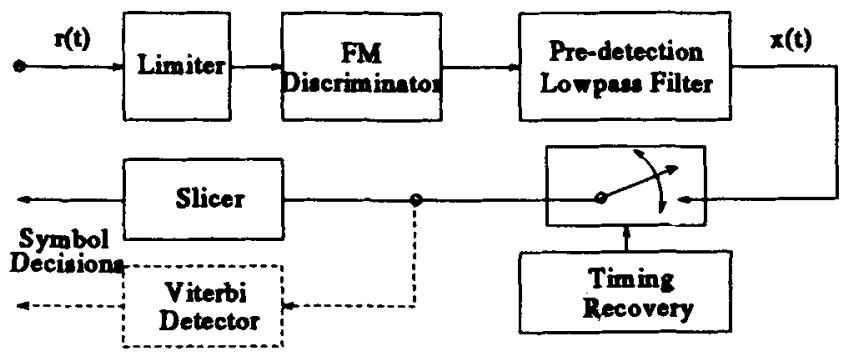

Figure 3. Digital FM demodulator

referred to as a digital FM detector (Pawula 1981) and it goes hand-in-hand with a VCObased modulator as shown in figure 1. This digital FM receiver is illustrated in figure 3, where $x(t)$ is the noisy baseband signal. Note that, in the absence of noise, the limiterdiscriminator output is the message signal $m(t)$ given in (3). The lowpass filter at the discriminator output must have a bandwidth greater than the bandwidth of the Gaussian filter so that $m(t)$ is not distorted. The remaining baseband processing tasks are: (a) clock recovery, and (b) data detection. In figure 3, the simple threshold detector (slicer) can be replaced by a matched filter or a Viterbi detector which implements Maximum Likelihood Sequence Estimation in order to improve the performance.

It is also known (Haykin 1983) that if the SNR at the discriminator input is above a certain threshold, the output of the discriminator can be taken as $m(t)$ with additive noise $n(t)$. The output noise spectral density $S_{n}(f)$ is of the form (Haykin 1983, ch. 6)

$$
S_{n}(f)=\left\{\begin{array}{cc}
N_{0} f^{2}, & -W<f<W, \\
0, & \text { otherwise }
\end{array}\right.
$$

where $2 W$ is the bandwidth of the bandpass filter at the limiter input and $N_{0} / 2$ is the spectral height of the AWGN at the demodulator input. Such a parabolic noise spectral density implies that the noise is correlated from one symbol-duration to the next.

\section{DSP-based digital FM detector}

Detection of the data from the baseband signal, $x(t)$, is accomplished by (a) recovering the bit clock, and (b) sampling and thresholding (slicing). The sampling/thresholding step may be preceded by matched-filtering. Even though the noise is not white at the matched-filter input, such filtering typically improves the performance of the detector. Matched-filtering is easier to implement when the baseband pulse is designed so as to prevent ISI at the matched filter output, e.g., a rectangular pulse of duration $T$. If the pulse is not so designed, as is the case with GMSK, matched filtering cannot be performed on a bit-by-bit basis. Rather, we need a match to the entire received sequence. This is accomplished by the so-called maximum likelihood sequence estimator which can be implemented efficiently using the Viterbi algorithm. MLSE-based data detection is discussed in $\S 4$.

In the present section, we focus our attention on the tasks of clock recovery and data detection, which are performed without using any filtering operation. Generally, such an approach lends itself very nicely to an all-hardware implementation. However, we propose a clock-recovery and data-detection algorithm based fully on discrete-time signal processing. While this DSP implementation is comparable in its complexity and performance to a hardware implementation, it becomes a more favourable approach when coupled with the more sophisticated MLSE-based detector. 


\subsection{Clock recovery}

In any modem, clock recovery is performed for two reasons: (a) to play out the detected data at exactly the same rate as is being transmitted, and (b) to assist the modem in performing as well as possible for any given performance criterion.

Let us consider specifically a digital FM receiver based on sampling and thresholding the baseband signal $x(t)$. We desire here to recover a clock signal such that when $x(t)$ is sampled and thresholded with respect to this clock, the bit-error probability, $P_{e}$, is as small as possible. When there is ISI in $x(t), P_{e}$ for a given bit may vary depending on the values of the bits occuring before and after it. For example, in GMSK with $B T=0.5$ or less, there is a significant amount of ISI. In such a case, one may wish to minimise either an average bit-error probability, $P_{e a}$, or the worst-case bit-error probability, $P_{e w}$, for the bit whose error probability is highest due to ISI. In either case, the recovered clock must be such that either $P_{e a}$ or $P_{e w}$ is minimised, as desired. In this paper, we choose $P_{e w}$ as the performance measure.

Let us assume, as is usual in most clock recovery schemes, that the clock frequency is known precisely, and only its phase is unknown. Frequency error, if any, is assumed to be so small that it manifests only as a slowly-changing clock phase, which can be tracked. Let $x_{\alpha}(n T)$ be the samples of $x(t)$ sampled according at $t=n T+\alpha$. The $n$th bit is thus decided by the sign of $x_{\alpha}(n T)$.

We desire to choose $\alpha$ such that $P_{e w}$ is minimised. Since the noise variance is independent of time, $P_{e w}$ is minimised when $\alpha$ is chosen so as to maximise $\min \left\{x_{\alpha}(n T)\right\}$. That is, the sampling clock is chosen such that the bit with the maximum ISI (and hence worst $P_{e}$ for a given noise variance) has the maximum amplitude at the sampling instant. For example, the optimum sampling instants for a GMSK signal with $B T=0.5$ are shown by cross-marks in figure 2.

\subsection{Tracking of clock phase}

The design of many modems include a training-sequence or preamble for enabling the receiver, among other things, to synchronise itself with the transmit clock phase. The maximum-likelihood estimator of clock phase for a known signal in white noise is the correlator (Ziemer \& Peterson 1985, ch.6). The correlator, whose output is maximum when the input is aligned with its own stored copy of the known signal, is often employed even when the noise is not white. In order to obtain the optimal clock-phase estimate that minimises $P_{e w}$, the preamble must be such that it has a correlation peak at the optimal time instant. This is achieved by using a preamble with an appropriately chosen periodic data pattern. An example is shown in figure 4 for a GMSK signal with $B T=0.5$. An alternating one-zero data pattern ensures that the correlator output will peak at the instants when $x(t)$ reaches its largest value in a bit-duration where the ISI is greatest.

In continuously-operating modems, after an initial training-period, the receiver tracks the slowly-varying clock phase. This tracking is performed using any of a number of wellknown techniques, such as the early-late method (Ziemer \& Peterson 1985, ch. 6). For the digital FM receiver, the tracking-loop error signal must be updated only for those bits with maximum ISI. In modems for bursty transmission, as in TDMA systems, usually every burst includes a preamble for estimating the clock phase. Further, since the clock phase will not vary significantly during the burst, no tracking is needed while detecting data in that burst. 


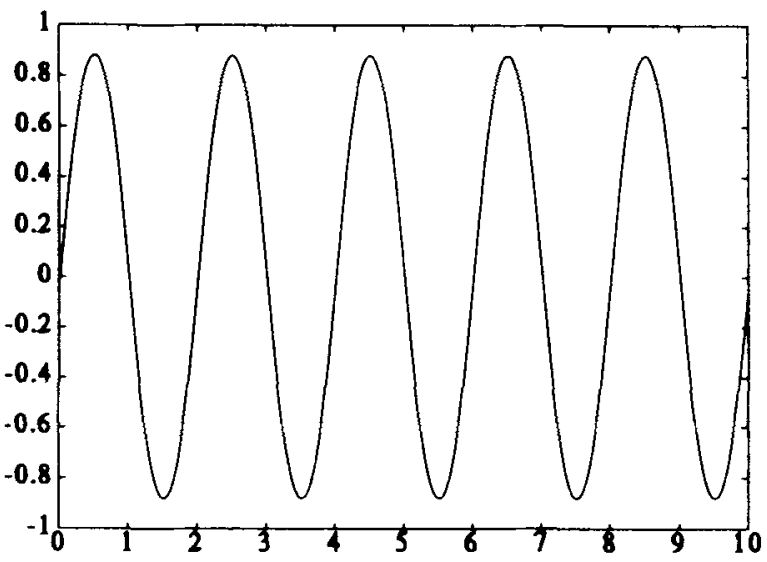

Figure 4. Alternating one-zero pattem for clock recovery

Phase-locked/tracking loops are conventional hardware implementations of the clock recovery techniques discussed above. We consider next a completely algorithmic DSPbased implementation but, prior to that, we discuss the sampling of $x(t)$ for discrete-time processing.

\subsection{Sampling of the baseband signal}

In most digital modulation schemes, the bandwidth of the baseband signal is usually some value in the range $[R / 2 . R]$ where $R$ is the symbol rate. In selecting the sampling rate, $f_{s}$, it is convenient to choose a multiple of the symbol rate that also satisfies the Nyquist criterion. Typically, a sampling rate of $2 T$ is sufficient when the bandwidth of $x(t)$ is as mentioned above. Most detection algorithms are executed once every symbol, and a sampling rate which is a multiple of $R$ simplifies the algorithm considerably.

In the case of GMSK, a sampling rate of $2 R$ is suitable for all values of the Gaussian filter bandwidth $B$, even if it is somewhat liberal when $B T$ is small. It is assumed, of course, that the noise is also bandlimited to a bandwidth less than $f_{s} / 2$. This is usually accomplished at the intermediate frequency (IF) stage, where the IF filter that provides the selectivity also bandlimits the noise.

\subsection{Clock recovery in discrete-time}

Let $x(t)$ be sampled at rate $f_{s}=1 / T=2 R=2 / T$, and let the samples be denoted $x\left(n T_{s}\right)$. Let $T_{r}=T_{s} / M$ be the time resolution with which we perform clock-phase estimation. Here, $M$ can be chosen as large as required; typically, values in the range $5 \sim 10$ suffice.

Clock-phase estimation amounts to determining which of the $M$ instants $T_{(n, i)}=n T_{s}+$ $i T_{r}, i=0,1,2, \ldots, M-1$, is the best sampling instant. In a burst modem, the optimum instant $T_{(n, j)}$ is determined at the beginning, and $j$ usually does not change during the burst (unless $M$ is large). In a continuously-operating modem, the clock is tracked and the value of $j$ in $T_{(n, j)}$ changes slowly with $n$.

Since we sample at a rate greater than the Nyquist rate, correlation of the preamble can be performed in discrete-time. The correlator output is interpolated at the $M-1$ instants $T_{(n, i)}=n T_{s}+i T_{r}, i=1,2, \ldots, M-1$ in between each output sample. For doing this task, $M-1$ interpolators are needed. The maximum of the interpolated samples, occurring say, at $T_{(n, j)}$, gives the value $j$ corresponding to the best sampling clock (for a time-resolution of $T_{r}$. The $j$ th interpolator is then employed to interpolate $x(t)$ at $t=n T_{s}+j T_{r}$, and the sign of the interpolated value indicates the data bit. 
Thus, clock recovery in discrete-time is tantamount to determining which of $M$ interpolators to employ, where $M$ determines the resolution of our estimate. If the clock phase is being tracked, the value of $j$ will change slowly, increasing or decreasing by unity every once in a while. When it reaches 0 (or $M$ ), a sample of the input $x\left(n T_{s}\right)$ is repeated (or dropped) in order to adjust the sampling rate to precisely $2 R$, where $R$ is the bit rate. The interpolator may be designed using any conventional approach (Vaidyanathan 1993).

\section{Digital FM Detection using the VA}

When digital FM detection is based on sampling once per bit and slicing, we are not exploiting the baseband signal over the entire bit duration. Although the noise is not white, and matched filtering is therefore not optimal, such filtering would nevertheless lead to some improvement in performance. However, matched filtering cannot be performed on a bit-by-bit basis when ISI is present at the matched filter input. In this case, an entire sequence of bits has to be matched to, i.e., we have to perform MLSE.

If the noise is white, the optimal MLSE of an $N$-bit data sequence is defined as follows (Proakis 1989, ch. 6). Let $r(t)$ be the received signal and $x^{(i)}(t), i=0,1, \ldots, 2^{N}-1$ be the transmitted signal for the $i$ th data sequence (of the $2^{N}$ possible $N$-bit sequences). We decide in favour of the $j$ th sequence if the Euclidean distance $D_{j}<D_{i}, \forall i \neq j$, where

$$
D_{i}=\int_{0}^{N T}\left[r(t)-x^{(i)}(t)\right]^{2} \mathrm{~d} t
$$

This distance can be computed equivalently from the sampled sequences $r\left(n T_{s}\right)$ and $x^{(j)}\left(n T_{s}\right)$ as follows:

$$
D_{i}=\sum_{n=0}^{2 N-1}\left[r\left(n T_{s}\right)-x^{(i)}\left(n T_{s}\right)\right]^{2} \mathrm{~d} t
$$

where we have assumed that there are two samples per bit. Note also that $D_{i}$ can be obtained from any set of uniformly spaced samples $r_{\alpha}\left(n T_{s}\right)=r\left(n T_{s}+\alpha\right)$ and $x_{\alpha}^{(i)}\left(n T_{s}\right)=$ $x^{(i)}\left(n T_{s}+\alpha\right)$ for any $\alpha \in\left[0, T_{s}\right]$, i.e.,

$$
\begin{aligned}
D_{i} & =\sum_{n=0}^{2 N-1}\left[r_{\alpha}\left(n T_{s}\right)-x_{\alpha}^{(i)}\left(n T_{s}\right)\right]^{2} \\
& =\sum_{k=0}^{N-1} \sum_{l=0}^{1}\left[r_{\alpha}\left((2 k+l) T_{s}\right)-x_{\alpha}^{(i)}\left((2 k+l) T_{s}\right)\right]^{2} .
\end{aligned}
$$

From the above we see that $D_{i}$ can be accumulated on a bit-by-bit basis, the inner sum (over $l$ ) being the metric for the $k$ th bit. Thus, determination of the minimum distance, $D_{j}$, can be performed using the Viterbi Algorithm (Proakis 1989). The sampled baseband signal $x^{(i)}\left(n T_{s}\right)$ is given by

$$
x^{(i)}\left(n T_{s}\right)=\sum_{k=0}^{N-1} I_{k}^{(i)} g\left(n T_{s}+\alpha-k T\right)
$$

where $\left\{I_{k}^{(i)}\right\}_{k=0}^{N-1}$ is the $N$-bit binary expansion of $i$ th sequence. Now, if $g(t)$ is non-zero in $\left[0, T_{g}\right]$, (and dropping the index $i$ for convenience), the even and odd samples in the $m$ th bit-duration are given by 
Time:

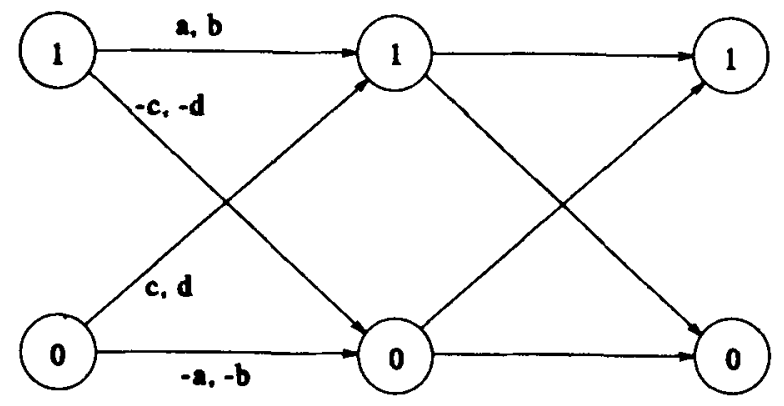

Figure 5. Trellis example for $L=2$ where: $\mathbf{a}=g(\alpha)+g\left(2 T_{s}+\alpha\right), \mathbf{b}=$ $g\left(T_{s}+\alpha\right)+g\left(3 T_{s}+\alpha\right), \mathbf{c}=g(\alpha)-$ $g\left(2 T_{s}+\alpha\right)$, and $\mathbf{d}=g\left(T_{s}+\alpha\right)-g\left(3 T_{s}+\right.$ $\alpha)$.

$$
x_{\alpha}\left(2 m T_{s}\right)=\sum_{k=m-L+1}^{m} I_{k} g\left(2(m-k) T_{s}+\alpha\right)
$$

and

$$
x_{\alpha}\left((2 m+1) T_{s}\right)=\sum_{k=m-L+1}^{m} I_{k} g\left(2(m-k) T_{s}+T_{s}+\alpha\right)
$$

respectively, where $L=\operatorname{ceil}\left(T_{g} / T\right)$ specifies the number of symbols contributing to the signal amplitude at the sampling instant. Here, the function ceil(.) rounds toward the upper integer. Depending on the value of $\alpha$, the symbol $I_{m-L+1}$ may or may not be included in one of the sums in (10) and (11). Thus, the number of states in the trellis is $2^{L-1}$. A state is labelled by the $L-1$ bits $\left\{I_{m-L+1}, \ldots, I_{m-1}\right.$. Two paths radiate from each state depending on the value of $I_{m}$, and each state is also entered by two paths. For example a trellis diagram for $L=2$ is shown in figure 5 .

There are two equivalent options for implementing the VA. In both cases, timing recovery is performed separately as discussed in $\S 3$. In the first option, the trellis is populated with samples according to (10) and (11) for a particular value of $\alpha$, say $\alpha=0$. Based on the estimate of $\alpha$ obtained from timing recovery, an appropriate interpolator is employed to interpolate the samples of $r(t)$ at $t=n T_{s}$. These interpolated samples are then employed in the VA. Note that both samples in each bit interval are to be interpolated. In the second option, $x\left(2 m T_{s}\right)$ and $x\left((2 m+1) T_{s}\right)$ are pre-computed for $M$ (where $M=T_{s} / T_{r}$, see $\S 3.4$ ), different values of $\alpha$ in $\left[0, T_{s}\right]$. Depending on the estimate of $\alpha$, the appropriate set of samples are used to populate the VA for the duration of time during which the estimate of $\alpha$ remains unchanged. The second option will save on computation as run-time interpolation of $r\left(n T_{s}\right)$ is avoided.

\subsection{Whitening filter for improved performance}

The MLSE is optimal only for signals in white Gaussian noise. If the noise is Gaussian but coloured, and the spectral density is strictly positive within the band of interest, then the optimal detector can be implemented as a cascade of a whitening filter and MLSE (Wozencraft \& Jacobs 1965 , ch. ?). The pulse shape $g(t)$ is replaced by $g_{w}(t)=g(t) \star h(t)$, where $h(t)$ is the impulse response of the whitening filter and $\star$ represents the convolution operation. Since the duration of $g_{w}(t)$ will, in general, be longer than that of $g(t)$, the number of states in the trellis may increase. The input $r\left(n T_{s}\right)$ is convolved with $h\left(n T_{s}\right)$, and the output is fed to the VA. The trellis is populated with samples of $x(t)$ as in (10) and (11), with $g($.$) replaced by g_{w}($.$) , and L$ now being determined by the duration of $g_{w}($.$) . Either$ option may be exercised in implementing the VA, as discussed above. 


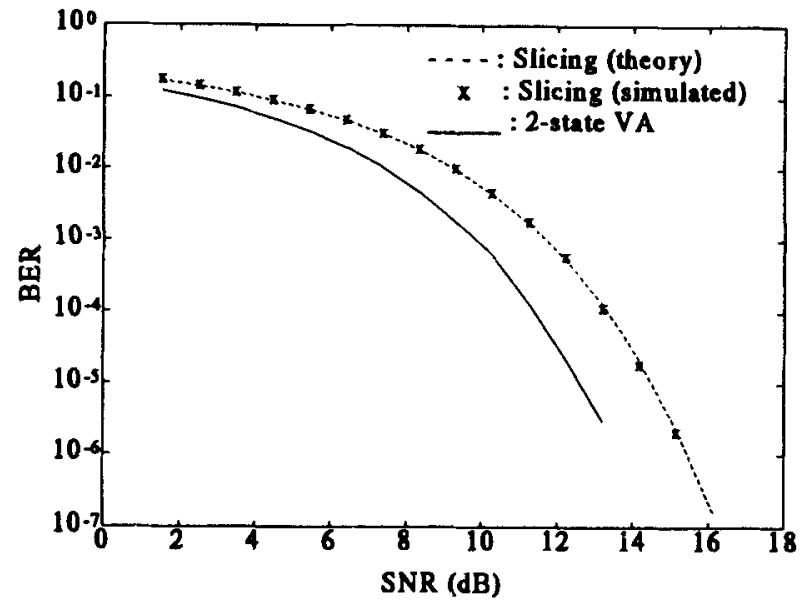

Figure 6. BERs for the slicer and Viterbi detector in white noise

In the case of digital FM, the noise spectral density is parabolic with a null at $f=0$. As such, this noise cannot be whitened exactly. Approximate whitening filters, which are essentially lowpass filters with a response proportional to $1 / f$ as $f$ increase from zero, can be employed. An FIR filter of low order is preferable so that the number of states in the trellis does not increase significantly.

\section{Simulation results and conclusions}

In this section, we compare the simulated BER performance of the digital FM demodulator for three detectors: slicer, sequence estimator without whitening filter, and MLSE with an approximate whitening filter. GMSK with $B T=0.5$ was chosen for the simulations. The impulse response of the Gaussian shaper, $g(t)$, was approximated to be time-limited to $[0,2 T]$ while generating the received samples. This implies that, even when perfect timing recovery leading to sampling (or interpolation) at the optimal instants is assumed, there still exists a 3\% ISI contribution from adjacent symbols $I_{k-1}$ and $I_{k+1}$ on the even-numbered sample corresponding to symbol $I_{k}$. Note that the ISI contribution on the odd-numbered sample of $I_{k}$ from $I_{k-1}$ equals about $50 \%$ of the signal amplitude.

In constructing the VA, we neglect the small ISI contribution to the even-numbered sample from symbol $I_{k+1}$, in order to retain a 2 -state trellis. It should also be mentioned here that when there is no whitening filter, a 2-state trellis can always be ensured (independent

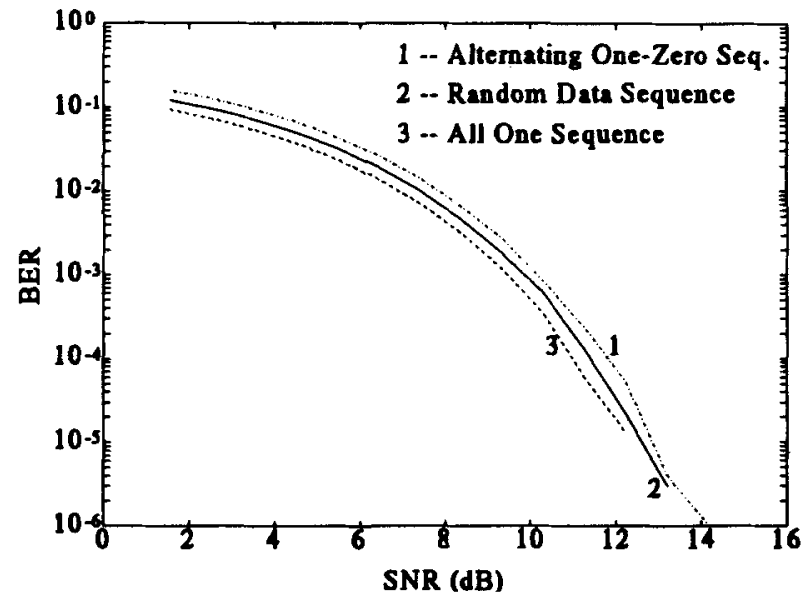

Figure 7. BERs for different data sequences in white noise 


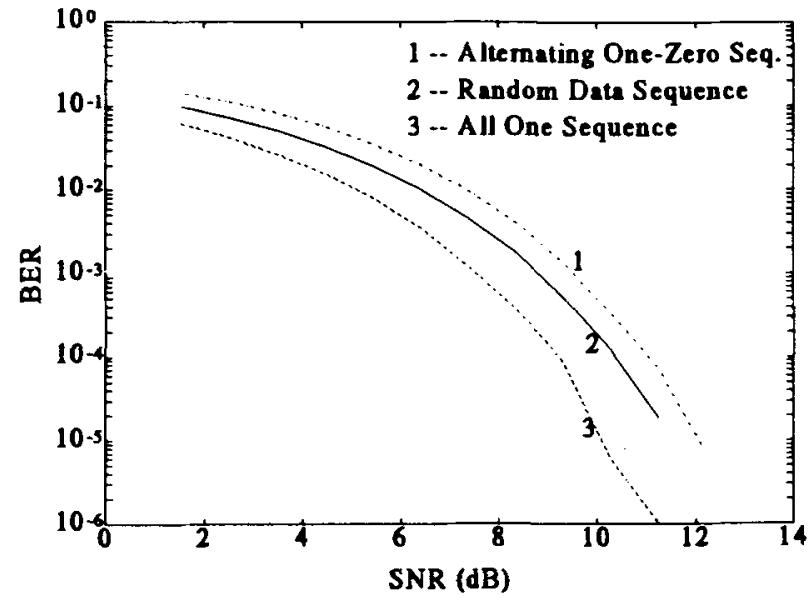

Figure 8. BERs for different data sequences in coloured noise

of the sampling instances) by correctly grouping the samples corresponding to the same bit interval. This is clear from (10) and (11). Either of the two options described in $\$ 4$ can be exercised in implementing the VA, where the option without run-time interpolation is more computationally efficient.

When a whitening filter is present, we know from (10) and (11) that the number of states in the trellis is determined by the duration of $g_{w}(t)$, the output of the whitening filter. Further, one of the samples in each bit duration may depend on only $L-2$ past bits, depending on the value of $\alpha$. The number of states in the trellis can differ by a factor of two, depending on $\alpha$ and also on whether the whitening filter order is odd or even. Thus, by employing interpolated sample values, it becomes possible to use a higher-order whitening filter for the same VA complexity. In other words, run-time interpolation helps in obtaining a superior BER performance for the same VA complexity, when compared to using the samples directly with a lower-order whitening filter. Therefore, a trade-off exists between the computational complexity of the VA and that of the run-time interpolator, when a whitening filter is introduced before the VA.

Figure 6 compares the BER of the slicer with that of a 2-state VA for AWGN. The AWGN case is reported because the result can be verified with theory. The theoretical BER curve for antipodal signalling, namely $(1 / 2) \operatorname{erfc}\left(E_{b} / N_{0}\right)^{1 / 2}$, where erfc (.) is the complementary error function, is also shown. For the 2 -state VA, at high SNRs, the BER is dominated by the single-bit error event, resulting in a Euclidean distance equal to $6 A_{m}^{2}$ approximately (where $A_{m}$ is the maximum amplitude during the bit). When compared to the slicer, which has a Euclidean distance of $4 A_{m}^{2}$, the VA thus has a $1.8 \mathrm{~dB}$ advantage in SNR. Indeed, this $1.8 \mathrm{~dB}$ gain is evident in figure 6 when the SNR is large.

Since the instantaneous bandwidth of the GMSK signal is a function of the actual bit sequence, we are interested in studying the performance of the sequence which contributes to the maximum deviation (the alternating one-zero pattern) and the one which contributes to the least (the all-one or all-zero pattern). The BER performance of these particular sequences are compared to that of the random (message) sequence in figure 7 , for VA in white noise. As expected, the performance of the random data sequence lies in between the performance for sequences which use the maximum and minimum channel bandwidth.

Figure 8 compares the BER for these three different data sequences in coloured Gaussian noise with a parabolic spectral density as in (5) for $-0.75 / T<f<0.75 / T$ and with a cosine roll-off till $\pm W$ (to approximate the roll-off due to the pre-detection low-pass filter). The total noise power was kept the same as in the white-noise case. The performance of 


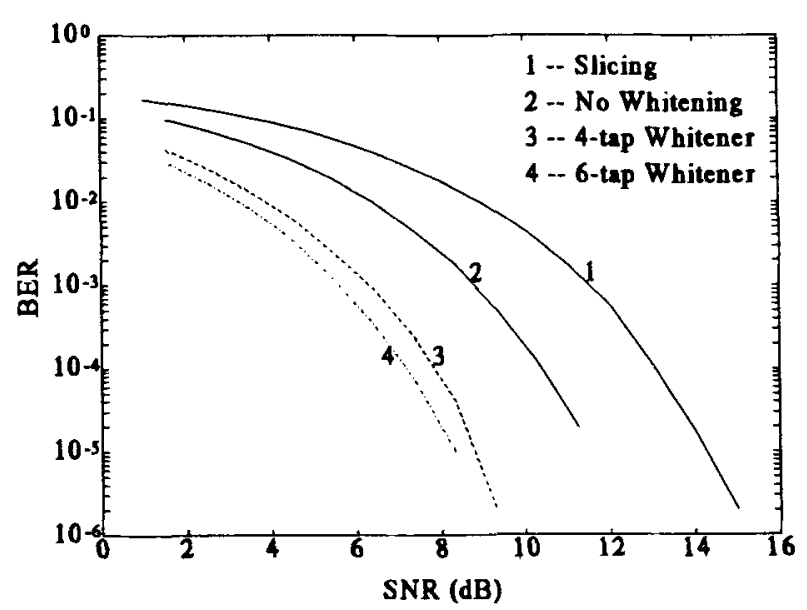

Figure 9. BER performance of the VA with whitening filter

the slicer is independent of the data sequence and noise spectral density, and is nearly identical to the result shown in figure 6. With the VA, we find that the BER improves in all cases when the noise is coloured. However, since the same noise power is distributed in a parabolic fashion, the low-frequency, all-one sequence experiences a greater reduction in BER with respect to the random sequence, when compared to the results for white noise.

Finally, figure 9 compares the performance of the VA when a 4-tap or 6-tap FIR filter is introduced to approximately whiten the coloured noise. The magnitude response of these filters were chosen based on a numerical search technique so that the noise PSD at the output is approximately flat over the bandwidth $-0.75 / T<f<0.75 / T$. While the resulting 4-tap whitener leads to a 4-state VA, the 6-tap whitener increases the number of VA states to eight. In both cases, the input samples were interpolated based on the recovered clock in order to minimise the number of states. The BER curves of the slicer and the 2-state VA for the coloured-noise case are also shown in figure 9.

Observe that even with a 4-tap whitener, about $5.5 \mathrm{~dB}$ gain in SNR (at BER $=10^{-4}$ ) is possible over the slicer. For an 8-state VA corresponding to a 6-tap whitener, an additional $0.8 \mathrm{~dB}$ gain is accrued. Increasing the whitening-filter order (and the VA complexity) further will yield only marginal SNR improvement.

The assumption of an additive-noise model with parabolic spectral density is valid only when the SNR at the demodulator input is above the threshold. This threshold varies between 10 and $14 \mathrm{~dB}$ (approx.) depending on the type of demodulator (I-Q or discriminator). At SNR levels near threshold or below, an additional "click-noise" component must be included in the model (Mazo \& Salz 1966). Work remains to be done for determining the performance of the VA-based detector when click-noise is also present.

Another interesting extension of this work is for the equalisation of ISI channels. Multipath propagation leading to fading and ISI is a common occurrence in mobile/personal communication systems. A model is needed for the FM-demodulated baseband signal when the received signal has multipath interference. Based on this model, the VA-based detector can be modified to include the effect of distortion of the baseband pulse. The authors are currently pursuing work along these directions.

\section{References}

ETSI 1992 Digital European Cordless telecommunications common interface Part 2: Physical layer, ETSI 300175.2 
GSM 1991 GSM recommendations 05.04, ver 3.1.2: Modulation

Haykin S 1983 Communication systems 2nd edn (New Delhi: Wiley Eastern)

Hirade K, Murota K 1981 GMSK modulation for digital mobile telephony. IEEE Trans. Commun. COM-29: 1044-1050

Mazo J E, Salz J 1966 Theory of error rates of digital FM. Bell Syst. Tech. J. 45: 1511-1535

Pawula R F 1981 On the theory of error rates for narrowband digital FM. IEEE Trans. Commun. COM-29: 1634-1642

Proakis J G 1989 Digital communications 2nd edn (New York: McGraw Hill)

Vaidyanathan P P 1993 Multirate systems and filter banks (Englewood Cliffs, NJ: Prentice Hall) Wozencraft J M, Jacobs I M 1965 Principles of communication engineering (New York: Wiley)

Ziemer R E, Peterson R L 1985 Digital communications and spread spectrum systems (New York: Macmillan) 